Smolders, M., Laurant, M., Verhaak, P., Prins, M., Marwijk, H. van, Penninx, B., Wensing, M., Grol, R. Which7 physician and practice characteristics are associated with adherence to evidence-based guidelines for $\mathrm{n}$ depressive and anxiety disorders? Medical Care: 2010, 48(3), 240-248

\begin{tabular}{|l|l|}
\hline Postprint Version & 1.0 \\
\hline Journal website & $\begin{array}{l}\text { http://journals.lww.com/lww- } \\
\text { medicalcare/pages/articleviewer.aspx?year=2010\&issue=03000\&article=00008\& }\end{array}$ \\
\hline $\begin{array}{l}\text { type=abstract } \\
\text { Pubmed link }\end{array}$ & $\underline{\underline{\text { http://www.ncbi.nlm.nih.gov/pubmed/20125045 }}}$ \\
\hline DOI & $10.1097 /$ MLR.0b013e3181ca27f6 \\
\hline
\end{tabular}

This is a NIVEL certified Post Print, more info at http://www.nivel.eu

\title{
Which Physician and Practice Characteristics are Associated With Adherence to Evidence-Based Guidelines for Depressive and Anxiety Disorders?
}

\author{
Smolders, Mirrian PhD*; LAURAnt, Miranda PHD*; VerhaAK, PETER PhD†; Prins, MARIJN \\ MSC†; VAN MARWIJK, HARM PHD + ; PENNINX, BRENDA PHD§đ|l; WENSING, MiCHEL PHD*; GROL, \\ RICHARD PHD*.
}

Author Information.

From the * Scientific Institute for Quality of Healthcare, Radboud University Nijmegen Medical Centre (RUNMC), Nijmegen, The Netherlands;

†NIVEL, Netherlands Institute for Health Services Research, Utrecht, The Netherlands; Departments of ¥General Practice, and

§Psychiatry, EMGO Institute, VU University Medical Center, Amsterdam, The Netherlands; IDepartment of Psychiatry, Leiden University Medical Centre, Leiden, The Netherlands; and ||Department of Psychiatry, University Medical Centre Groningen, Groningen, The Netherlands.

The infrastructure for the NESDA study (www.nesda.nl) is funded through the Geestkracht program of the Netherlands Organization for Health Research and Development (ZON-MW, grant number 10-000-1002) and is supported by participating universities and mental health care organizations (VU University Medical Center, GGZ inGeest, Arkin, Leiden University Medical Center, GGZ Rivierduinen, University Medical Center Groningen, Lentis, GGZ Friesland, GGZ Drenthe, Scientific Institute for Quality of Healthcare [IQ healthcare], Netherlands Institute for Health Services Research [NIVEL], and Netherlands Institute of Mental Health and Addition [Trimbos]). Financial support for data analyses for the present study was provided by ZonMw (grant number 945-14-413).

Reprints: Mirrian Smolders, PhD, IQ healthcare, Radboud University Nijmegen Medical Centre, PO Box 9101; code 114 IQ healthcare, 6500 HB, Nijmegen, The Netherlands. E-mail: m.smolders@iq.umcn.nl.

\begin{abstract}
.
Background: Research on quality of care for depressive and anxiety disorders has reported low rates of adherence to evidence-based depression and anxiety guidelines. To improve this care, we need a better understanding of the factors determining guideline adherence.

Objective: To investigate how practice- and professional-related factors are associated with adherence to these guidelines.

Design: Cross-sectional cohort study.

Participants: A total of 665 patients with a composite interview diagnostic instrument diagnosis of depressive or anxiety disorders, and 62 general practitioners from 21 practices participated.

Measures: Actual care data were derived from electronic medical record data. The measurement of guideline adherence was based on performance indicators derived from evidence-based guidelines. Practice-, professional-, and patient-related characteristics were measured with questionnaires. The characteristics associated with guideline adherence were assessed by multivariate multilevel regression analysis.
\end{abstract}


Smolders, M., Laurant, M., Verhaak, P., Prins, M., Marwijk, H. van, Penninx, B., Wensing, M., Grol, R. Which physician and practice characteristics are associated with adherence to evidence-based guidelines for depressive and anxiety disorders? Medical Care: 2010, 48(3), 240-248

Results: A number of practice and professional characteristics showed a significant univariate association with guideline adherence. The multivariate multilevel analyses revealed that, after controlling for patient characteristics, higher rates of guideline adherence were associated with stronger confidence in depression identification, less perceived time limitations, and less perceived barriers for guideline implementation. These professional-related determinants differed among the overall concept of guideline adherence and the various treatment options.

Conclusions: This study showed that rates of adherence to guidelines on depressive and anxiety disorders were not associated with practice characteristics, but to some extent with physician characteristics. Although most of the identified professional-related determinants are very difficult to change, our results give some directions for improving depression and anxiety care.

Depressive and anxiety disorders are highly prevalent conditions which are known to cause a wide range of disabilities and impairments, and which have considerable economic consequences as well.1-3 Depressive and anxiety disorders are the most common mental disorders with lifetime prevalences of $19.0 \%$ and $19.3 \%$, respectively. 1 A substantial number of national and international evidence-based guidelines for the management of depressive and anxiety disorders have been issued during the last several years. ${ }^{4-7}$ Nonpharmacological therapies, such as psychologic support and counseling, and pharmacological therapies, such as antidepressant medication, are now recommended for treatment.

Additionally, referral of a patient to primary or secondary specialized mental health care is an adequate option for the management of depressive and anxiety disorders.

Research on quality of care for depressive and anxiety disorders has reported low rates of guideline adherence. ${ }^{8-14}$ Although it is generally considered legitimate to deviate from guideline-recommended care under particular circumstances, noncompliance usually has significant health consequences as treatment meeting clinical guidelines decreases years lived with disability, and is cost-effective. ${ }^{15}$ Improving guideline adherence is a complex issue as it is influenced by individual characteristics of both the physician and the patient, and also by characteristics related to the organizational context. ${ }^{16}$ In earlier studies on adherence to guidelines, it was revealed that physician characteristics that are associated with adherence to clinical practice guidelines for cancer screening include gender and age ${ }^{17}$ Furthermore, attitudes towards illness, treatment, and clinical practice guidelines seemed to influence adherence to both guidelines on diabetes care and smoking cessation guidelines. ${ }^{18,19}$ Several studies have examined patient factors associated with the provision of guideline-recommended care and have found that having more than one comorbid condition ${ }^{20}$ as well as patients' fear of and unwillingness to tolerate procedures ${ }^{21,22}$ are negatively associated with guideline adherence. Furthermore, previous research has demonstrated that organizational characteristics including practice size and location are associated with guideline adherence. ${ }^{23}$ By understanding which factors are associated with adherence to evidence-based guidelines on the management of depressive and anxiety disorders, strategies can be identified to improve physician guideline adherence and to adapt and focus guidelines, and thus improve the quality of depression and anxiety care. The current literature on determinants of adherence to evidence-based guidelines on depressive and anxiety disorders mainly focused on socio-demographic patient characteristics and disease-related factors, such as patient age and severity of the disorder. ${ }^{9,10,13}$ Despite its potential importance, prior studies did not examine the influence of professional- and practice-related factors on adherence to depression and anxiety guidelines. Our study therefore aimed to investigate how such factors interact in determining health care provision according to current primary care guidelines for the management of depressive and anxiety disorders. We hypothesized that the provision of guideline-recommended care will not merely be influenced by patient characteristics, but also by characteristics of both the professional and the practice. Furthermore, we hypothesized that these determinants will differ among the various guideline-recommended treatment options that are available for the management of depressive and anxiety disorders. 
Smolders, M., Laurant, M., Verhaak, P., Prins, M., Marwijk, H. van, Penninx, B., Wensing, M., Grol, R. Which physician and practice characteristics are associated with adherence to evidence-based guidelines for depressive and anxiety disorders? Medical Care: 2010, 48(3), 240-248

\section{METHODS.}

\section{Study Population and Setting.}

Data for this study came from the Netherlands Study of Depression and Anxiety (NESDA), an 8-year longitudinal cohort study designed to be representative of persons with depressive and anxiety disorders in different health care settings and in different stages of the disorders. Details on objectives, recruitment, and methods of NESDA have been described elsewhere. ${ }^{24}$ In short, recruitment of participants took place from September 2004 to February 2007. Seventy-two general practitioners (GPs) were invited to participate in the study. In selecting these GPs, attention was paid to the use of appropriate electronic patient record databases which allows uniform data extraction for research purposes. A random sample of 23,750 patients (aged 18-65 years), registered with those GPs, who attended their doctor in the last 4 months were sent a screening questionnaire to measure the presence of affective or anxiety disorders. Nearly half of the screeners returned were screen-positive and these persons were interviewed by phone with the short form of the composite interview diagnostic instrument (CIDI). ${ }^{25}$ If this interview produced a current (6-month recency) depressive disorder (including major depressive disorder or dysthymia) or anxiety disorder (including generalized anxiety disorder, social phobia, agoraphobia, or panic disorder), respondents were asked to participate in NESDA and were invited for a baseline assessment, which included a full CIDI interview. In addition, a random selection of the screen-negatives (both from the written screener or the phone screen) was also invited to participate (control group). Figure 1 depicts the screening procedure used for the recruitment of NESDA respondents in primary care. The NESDA study received ethical approval from the Central Ethics Committee on Research involving human subjects of the VU University Medical Center, Amsterdam, The Netherlands.

\section{MEASURES.}

\section{Dependent Variables.}

The Dutch primary care guidelines for the management of depressive and anxiety disorders ${ }^{6,7}$ were rigorously developed according to principles of evidence-based medicine and were published in the Dutch scientific journal for GPs, which reaches about $85 \%$ of practitioners. ${ }^{26}$ Furthermore, they were disseminated via educational materials and quality improvement meetings. According to the Dutch primary care guidelines, ${ }^{6,7}$ several options are available in the management of depressive and anxiety disorders. GPs have nonpharmacological (psychologic support, counseling) and pharmacological (prescription of antidepressant medication) management options. Alternatively, they can refer patients to mental health specialists in primary or secondary health care.

In this study, the dependent variables were ( $a$ ) overall adherence to evidence-based guidelines on depressive and anxiety disorders, $(b)$ appropriate nonpharmacological treatment by the GP (ie, psychologic support or counseling), (c) appropriate pharmacological treatment by the GP, and ( $d$ ) referral by the GP to a mental health specialist.

These dependent variables - performance indicators - were derived from the Dutch evidence-based guidelines for the management of depressive and anxiety disorders in general practice. ${ }^{6,7}$ Overall adherence to the guideline on depressive disorders was defined as receiving appropriate psychologic support, or receiving counseling, or receiving appropriate antidepressant medication, or a referral to a mental health specialist in primary or secondary health care.

The sole criterion for appropriate psychologic support was the provision of at least $5 \mathrm{GP}$ consultations in the 15-week period after documentation of the International Classification of Primary Care code ${ }^{27}$ of depression or anxiety. Criteria for appropriate antidepressant medication were (a) prescription of antidepressant medication, $(b)$ the provision of a consultation by the GP within 6 weeks of initiating antidepressant drug treatment, and (c) continuation of antidepressant drug treatment for at least 5 months or cessation of antidepressant drug treatment after 2 to 6 weeks in case of no response.

\section{[FIGURE 1]}

Neither counseling nor referral included any additional conditions for appropriate care. The definition of adherence to the anxiety guideline was identical to that of adherence to the depression guideline, with the exception of the element of counseling. In accordance with the Dutch anxiety guideline for GPs, ${ }^{6}$ counseling was not included in the definition of adherence to the anxiety guideline. 
Smolders, M., Laurant, M., Verhaak, P., Prins, M., Marwijk, H. van, Penninx, B., Wensing, M., Grol, R. Which physician and practice characteristics are associated with adherence to evidence-based guidelines for depressive and anxiety disorders? Medical Care: 2010, 48(3), 240-248

Information on the provision of psychologic support, antidepressant treatment, and referral was gathered from the electronic medical patient records (EMRs). As the EMRs did not contain information on the provision of counseling, this information was obtained from patient-reported answers to specific questions included in the Perceived Need for Care Questionnaire. ${ }^{28}$

\section{Independent Variables.}

\section{Practice Characteristics..}

All participating patients were registered with a general practice. We collected data about a number of characteristics of these practices. Practice assistants or practice nurses were requested to complete a short questionnaire on practice characteristics.

We obtained practice characteristics from frequently used questions included in the visit instrument to assess practice management of van den Hombergh et al. ${ }^{29}$ The practice characteristics included practice type, number of GPs in the practice, collaboration with other health care professionals working in the practice, and size of practice population.

\section{Professional Characteristics.}

We collected the professional characteristics from the individual self-administered questionnaires sent to all GPs who were involved in the care of the participating primary care patients suffering from a depressive or anxiety disorder.

The questionnaire contained 6 parts.

The first part included demographic data: age, gender, clinical experience, and employment status of the GP. In addition, this part of the questionnaire contained one item on the trainer status of the GP, ie, whether the GP was an approved clinical supervisor and mentor of GP registrars.

In part 2, we measured the GP's interest and attitudes towards depressive and anxiety disorders. GPs' attitudes towards depressive and anxiety disorders were measured with the Depression Attitude Questionnaire (DAQ) ${ }^{30}$ and with the REASON. ${ }^{31}$ The DAQ is a self-completion instrument with statements to which respondents indicate strength of agreement or disagreement, in terms of their daily clinical experience. ${ }^{32}$ Previous factor analyses of DAQ findings have provided a 4-factor solution: treatment attitudes, professional unease, depression malleability, and depression identification.

The REASON measures GPs' attitudes to their role in the management of patients with depressive and anxiety disorders. The REASON consists of 17 questions measuring 2 constructs: professional comfort and competence with the care of mental health disorders and GPs' concerns about difficulties with the health care system in this area.

In addition, this part of the questionnaire contained a question on whether the GP had a special interest in patients with depressive or anxiety disorders (yes/no), and an item that assessed the amount of time (in hours) spent on education on depressive and anxiety disorders during the last 12 months. ${ }^{29}$ Part 3 included questions to assess "barriers to health care provision to patients with depressive or anxiety disorders." Two items measured whether time limitations, respectively, lack of knowledge and skills were perceived barriers to the provision of depression and anxiety care.

In part 4, we measured collaboration with professionals and institutions specialized in mental health care. Four dichotomous questions (yes/no) measured the collaboration of the GP with social workers, psychologists in primary care, mental health nurses in general practice, and institutions specialized in mental health care. The percentage of GPs reporting collaboration with at least one professional or institution specialized in mental health care was calculated.

Part 5 included questions to assess "perceived barriers for implementation of the depression and anxiety guidelines." This part contained 13 items, based on a validated questionnaire,${ }^{33}$ measuring barriers for implementation of evidencebased guidelines. A total sum score was calculated by summing the scores of all items.

In part 6 of our questionnaire, we measured GPs' levels of burnout. For measuring the burnout symptoms exhaustion, cynicism, and feelings of reduced competence, we used the Utrecht Burn-Out Scale for the contactual professions (UBOS-C). The UBOS-C includes 20 items divided into 3 subscales: emotional exhaustion, depersonalization, and personal accomplishment. Mean scores for these 3 components of burnout were calculated for each GP, taking into account the maximum allowed number of missing items. For each subscale, GPs were classified in 3 groups, referring to low, middle, and high scores on the 
Smolders, M., Laurant, M., Verhaak, P., Prins, M., Marwijk, H. van, Penninx, B., Wensing, M., Grol, R. Which physician and practice characteristics are associated with adherence to evidence-based guidelines for depressive and anxiety disorders? Medical Care: 2010, 48(3), 240-248

subscale. Cut-off points for low, middle, and high levels of burnout on the subscales were derived from the group norms for Dutch primary care providers $(\mathrm{n}=1523)$, as published in the manual of the UBOS. ${ }^{34}$

\section{Control Variables.}

Several patient characteristics have been suggested to facilitate or impede guideline adherence. Previous research showed that appropriate treatment was less likely for men and those who were black, less educated, or younger than 30 or older than 59 years. ${ }^{13}$ Other studies have confirmed most of these determinants, as well as reported some other predictors of receiving guideline-concordant care, ie, being severely ill and suffering from comorbid conditions. ${ }^{9,10}$ As univariate analyses of our data showed that severity of symptomatology, gender, and age were significantly related to guideline adherence, these patient characteristics served as control variables in our analyses. Other patient characteristics, such as marital status, education, income, and comorbidity, were excluded as control variables because univariate analyses did not reveal a significant relation between those patient characteristics and adherence to evidence-based guidelines on depressive and anxiety disorders.

All patients, irrespective of their CIDI results, received questionnaires for measuring severity of symptomatology.

Severity of depressive symptoms was measured with the 30-item Inventory of Depressive Symptomatology self-report version. ${ }^{35}$ Severity of anxiety symptoms was measured using the 21 -item Beck Anxiety Inventory. ${ }^{36}$ Detailed sociodemographic and socioeconomic information was obtained through various questionnaires.

\section{STATISTICAL ANALYSIS.}

Descriptive statistics were used to outline the characteristics of practices, GPs, and patients. Only data from patients registered with GPs that responded to our questionnaire were included in our statistical analyses. Consistent with established guidelines and prior research studies in which the above mentioned instruments were used, subscale and total scores were calculated. Univariate and multivariate multilevel logistic regression analyses were performed first to identify determinants of the overall concept of guideline adherence, and subsequently to identify determinants of different treatment options (ie, appropriate nonpharmacological treatment, appropriate pharmacological treatment, and referral).

First, we investigated which practice and professional characteristics were related to overall guideline adherence, and to explore the relation with appropriate nonpharmacological treatment, appropriate pharmacological treatment and referral to a mental health specialist. Therefore, univariate associations between our 4 dependent variables and all the distinguished independent variables were computed, controlling for severity of symptomatology, patient age, and gender.

The variables that were found to be suitable for further analyses $(P \leq 0.10)$ were entered into a multivariate multilevel regression model, together with the control variables.

All independent variables with $P \leq 0.05$ and the control variables were retained in the final multivariate multilevel regression models. The dependent variables were the overall concept of guideline adherence and the various treatment options. As a correlation was detected between severity of depressive symptoms and severity of anxiety symptoms (correlation coefficient $>0.6$ ), we did not include both variables in the final regression models. Separate models were run, one including gender, age, and severity of depressive symptoms (Inventory of Depressive Symptomatology) as control variables and the other one including gender, age, and severity of anxiety symptoms (Beck Anxiety Inventory) as control variables.

As this yielded comparable results, we decided to present only the results of the model that included severity of depressive symptoms in the final multivariate multilevel regression analyses. Because of the hierarchical structure of our study (patients nested within practice), we took account of the variability associated with each level of nesting. We performed a model with a random intercept and all other variables fixed.

We chose to quantify the variance explained using a threshold model approach. ${ }^{37}$ Data were analyzed using SPSS version 14.0 and SAS version 9.2 (PROC GLIMMIX). 
Smolders, M., Laurant, M., Verhaak, P., Prins, M., Marwijk, H. van, Penninx, B., Wensing, M., Grol, R. Which physician and practice characteristics are associated with adherence to evidence-based guidelines for depressive and anxiety disorders? Medical Care: 2010, 48(3), 240-248

\section{[TABLE 1 AND TABLE 2].}

\section{RESULTS.}

\section{Study Population.}

Of the 1610 primary care patients included in NESDA, 743 patients met criteria on the full CIDI interview for either current (6-month recency) depressive disorder or current anxiety disorder. A total of 721 patients (97\%) gave informed consent for the extraction of health care information from their EMR. Of the 67 GPs involved in the care of these patients, 62 returned the questionnaire (response rate of 93\%). All questionnaires on practice characteristics were returned completed $(n=21)$. Six hundred sixty-five patients who gave informed consent were registered with a GP who returned the questionnaire.

Table 1 shows both demographic and treatment characteristics of the study population. One out of 5 patients suffered from a depressive disorder (without a comorbid anxiety diagnosis), whereas well over $40 \%$ of the patients had an anxiety disorder (without a comorbid diagnosis of a depressive disorder). Nearly $40 \%$ of the patients suffered from comorbid depressive and anxiety disorders. The mean age of the patients was 44.9 years. Less than one-third of the participants were male patients. Forty percent of the patients with a CIDI diagnosis of a current depressive or anxiety disorder was treated in accordance with the guidelines.

Among patients suffering from a current depressive or anxiety disorder, $16.1 \%$ received any form of appropriate nonpharmacological treatment, whereas nearly $19 \%$ was provided appropriate pharmacological treatment. Almost onequarter of the patients with a CIDI diagnosis of a current depressive or anxiety disorder was referred to specialized mental health care by the GP.

\section{Practice Characteristics.}

More than half of the practices were group practices (Table 2). The other ones were solo practices (14.3\%) or health centers $(28.6 \%)$. The mean size of the practice population was 5013 patients. About one-quarter of the practices reported the presence of a social worker or a psychologist in their practice.

\section{General Practitioners' Characteristics.}

Table 3 outlines characteristics of GPs. The mean age of the GPs was 48.8 years, and $54.8 \%$ of the GPs were men.

Nearly $40 \%$ of the GPs reported a special interest in patients with depressive and anxiety disorders. Half of the GPs experienced time limitations as a barrier to health care provision to patients with depressive and anxiety disorders.

Most of the GPs worked closely together with professionals or institutions specialized in mental health care. Collaboration between GPs and mental health nurses was mentioned most frequently.

Scores on the UBOS demonstrated that $6.5 \%$ of the GPs experienced high levels of emotional exhaustion, indicating serious symptoms of fatigue at work. Strong negative, cynical attitudes towards patients (depersonalization) were present in $6.5 \%$ of the GPs. Finally, $14.7 \%$ of the GPs experienced a lack of professional efficacy (personal accomplishment), whereas moderate to high levels of personal accomplishment were found in $47.5 \%$ and $37.7 \%$ of the GPs, respectively.

\section{Factors Associated With Adherence to Evidence-Based Guidelines on Depressive and Anxiety Disorders.}

Table 4 shows the univariate and multivariate associations between practice and professional characteristics on the one hand, and overall guideline adherence and the different treatment options on the other.

\section{Factors Associated With Overall Guideline Adherence.}

None of the practice-related characteristics showed sufficient association to be included in the multivariate regression model.

[table 3].

Results of the univariate analyses for associations between professional characteristics and overall guideline adherence indicated, however, that GPs with a strong confidence in their abilities to identify depression (DAQ component 4) as well as GPs who collaborated with professionals and institutions specialized in mental health care treated their patients more often in accordance with the guidelines than GPs who had difficulties with distinguishing depression from unhappiness and those who did not collaborate with mental health specialists, respectively. In addition, older GPs and GPs who perceived more barriers for guideline 
Smolders, M., Laurant, M., Verhaak, P., Prins, M., Marwijk, H. van, Penninx, B., Wensing, M., Grol, R. Which physician and practice characteristics are associated with adherence to evidence-based guidelines for depressive and anxiety disorders? Medical Care: 2010, 48(3), 240-248

implementation were less likely to adhere to the depression and anxiety guidelines compared with their younger colleagues and those who experienced less barriers for guideline implementation.

Only depression identification was retained in the final regression model, which demonstrated that this variable significantly influenced overall guideline adherence. Depression identification explained less than $1 \%$ of the variance in overall guideline adherence.

\section{Factors Associated With Appropriate Nonpharmacological Treatment.}

The univariate results indicated that none of the practice- related characteristics was related to the provision of appropriate nonpharmacological treatment.

At the level of the individual GP, however, univariate analyses showed that GPs who had more confidence in their own abilities to identify depression (DAQ component 4) as well as GPs with a stronger sense of personal efficacy (UBOS component 3) were more likely to provide appropriate nonpharmacological treatment compared with GPs with less confidence in their depression identification skills and GPs with feelings of low personal accomplishment, respectively.

Contrarily, results of univariate regression analyses showed that both perceiving concerns about difficulties with the health care system (REASON component 2) and perceiving time limitations diminished the probability of delivering appropriate nonpharmacological treatment by the GP.

Only one professional-related characteristic, ie, time limitations, was retained in the final multivariate multilevel regression model. The final model showed that perceived time limitations decreased the probability of delivering appropriate nonpharmacological treatment by the GP. Perceived time limitations explained less than $1 \%$ of the variance in appropriate nonpharmacological treatment.

\section{Factors Associated With Appropriate Pharmacological Treatment.}

Results of the univariate regression analyses showed that GPs working in practices with a small practice population tended to provide their patients appropriate pharmacological treatment more frequently compared with their colleagues with a large practice population. On the other hand, GPs who worked (nearly) fulltime were less likely to provide their patients appropriate pharmacological treatment compared with GPs who worked less hours a week..

[table 4].

Neither practice- nor professional-related characteristics remained significant in the multivariate regression analyses.

\section{Factors Associated With Referral to Specialized Mental Health Care.}

Univariate analyses did not reveal any associations between referral and practice characteristics.

Furthermore, univariate analyses showed that older GPs tended to refer their patients to specialized mental health care more frequently than their younger colleagues did. Also, GPs who perceived more barriers for guideline implementation referred their patients more often to mental health specialists than GPs who experienced these barriers to a lesser extent. Furthermore, univariate analyses showed that strong confidence of GPs in their abilities to identify depression (DAQ component 4), GPs' special interest in patients with depressive and anxiety disorders, and strong feelings of personal accomplishment by GPs (UBOS component 3 ) all decreased the probability of being referred to specialized mental health care by the GP.

Depression identification and perceived barriers for guideline implementation were retained in the final multivariate multilevel regression model. This model showed that both characteristics have a significant influence on referral to specialized mental health care. Depression identification and perceived barriers for guideline implementation explained less than $1 \%$ of the variance in referral to specialized mental health care.

\section{DISCUSSION.}

When included in a multivariate model, depression identification, perceived time limitations, and perceived barriers for guideline implementation proved to influence the overall concept of guideline adherence or one of the treatment options. GPs who had less confidence in their own abilities to identify depression were more likely to refer their patients to specialized mental health care. This characteristic was also positively associated with overall guideline adherence. Furthermore, GPs who experienced more barriers for guideline implementation were more inclined to refer their patients to mental health specialists. Finally, perceived time limitations decreased the probability of delivering appropriate nonpharmacological treatment. 
Smolders, M., Laurant, M., Verhaak, P., Prins, M., Marwijk, H. van, Penninx, B., Wensing, M., Grol, R. Which7 physician and practice characteristics are associated with adherence to evidence-based guidelines for depressive and anxiety disorders? Medical Care: 2010, 48(3), 240-248

Lack of time was also reported in other studies as a main barrier for guideline adherence. ${ }^{16,38}$ Unexpectedly, we found no relationship between collaboration of the GP with specialists in mental health care and guideline adherence.

Previous studies demonstrated, however, that collaborative care models for depressive and anxiety disorders improve the management and outcome of these disorders. ${ }^{39-42}$ An explanation for the apparent discrepancy between our study and previous ones could be that our outcome measure was not sufficiently sensitive to the influence of organization of care.

The various professional-related characteristics explained hardly any of the variance in guideline adherence or the various treatment options. This implies that other factors may play an important role. M Prins et al (unpublished data, 2009) found that patient characteristics which seem to play a role in adherence to evidence-based depression and anxiety guidelines are as follows: education level, evaluation of mental health care provider's behavior, and perceived need for care. Although the percentage of explained variance was exceptionally low in our study, a recent review also concluded that studies studying the link between organizational factors and quality outcomes in health care in general "are plagued with nonsignificant outcomes." 43

\section{Limitation and Strengths.}

A limitation of the present study was its cross-sectional design. Owing to this design, associations can be demonstrated, however, causal relationships cannot be inferred.

This limitation not withstanding, our study had the following strengths. First, our approach to assess guideline adherence was mainly prospective as we used primarily EMR data. Much of the literature in this area has relied on crosssectional interview data to assess guideline adherence. By employing a prospective design, we avoided problems associated with recall bias. Another strength of the present study was that it had a high response rate (93\% and $100 \%$ to GP questionnaires and practice questionnaires, respectively).

\section{PRACTICE IMPLICATIONS.}

Overall, our results may have implications for initiatives to optimize depression and anxiety care and to implement guidelines. Although time and resources for quality improvement activities are generally limited, tailored interventions to address specific barriers to physician guideline adherence may be most effective and efficient. ${ }^{44,45}$ Experiments with hands-on nursing staff are now taking place in Dutch primary care, that have demonstrated good results in other countries. ${ }^{46}$ Integrated nursing staff has also been a strong stimulus in other primary care quality improvement areas such as diabetes, asthma, and cardiovascular risk management.

${ }^{47,48}$ Such initiatives add time and resources for quality improvement activities. There is evidence that programs in which nurses play a vital role work. ${ }^{49,50}$ Although we assume that most patients can discuss their distress with the GP, many may benefit from a proper diagnostic assessment of potential psychopathology, or at least active monitoring. Previous research showed that overall guideline adherence was significantly better when depressive and/or anxiety disorders were recognized as such, compared with cases in which the depression or anxiety diagnosis was not made ( $74 \%$ vs. $33 \%$, respectively). ${ }^{14}$ The structured use of symptom questionnaires might be useful in increasing the GP's confidence in their skills to identify depressive and anxiety disorders. There are several, easy to complete, measures which have robust psychometric properties, for example the Four-Dimensional Symptom Questionnaire. ${ }^{51}$ The use of such questionnaires will improve the recognition rates and the eventual outcome of depressive and anxiety disorders in primary care. Implementation programs should facilitate acknowledgment of distress in general practice and offer GPs a range of tools to distinguish depression from unhappiness, and to deal with their many competing demands. ${ }^{52}$

\section{ACKNOWLEDGMENTS.}

The authors thank Stasja Draisma, Margot de Waal, and Willem Jan van der Veen for their help with data collection, and Reinier Akkermans for performing the multilevel analyses and his statistical advice.

\section{REFERENCES.}

1. Bijl RV, Ravelli A, van Zessen G. Prevalence of psychiatric disorder in the general population: results of The Netherlands Mental Health Survey and Incidence Study (NEMESIS). Soc Psychiatry Psychiatr Epidemiol. 1998;33:587-595. 
Smolders, M., Laurant, M., Verhaak, P., Prins, M., Marwijk, H. van, Penninx, B., Wensing, M., Grol, R. Which7 physician and practice characteristics are associated with adherence to evidence-based guidelines for 1 ( depressive and anxiety disorders? Medical Care: 2010, 48(3), 240-248

2. Lepine JP. Epidemiology, burden, and disability in depression and anxiety. J Clin Psychiatry. 2001;62(suppl 13):4-10.

3. Simon G, Ormel J, VonKorff M, et al. Health care costs associated with depressive and anxiety disorders in primary care. Am J Psychiatry. 1995;152:352-357.

4. National Institute for Clinical Excellence. Depression: Management of Depression in Primary and Secondary Care. Clinical Guideline 23. London, United Kingdom: National Institute for Clinical Excellence; 2004.

5. National Institute for Clinical Excellence. Anxiety: Management of Anxiety (Panic Disorder, With or Without Agoraphobia, and Generalised Anxiety Disorder) in Adults in Primary, Secondary and Community Care. Clinical Guideline 22. London, United Kingdom: National Institute for Clinical Excellence; 2004.

6. Terluin B, van Heest FB, van der Meer K, et al. Guideline anxiety disorders Dutch college of general practitioners (first revision). Huisarts Wet. 2004;47:26 -37.

7. van Marwijk HW, Grundmeijer HG, Bijl D, et al. Guideline depressive disorder Dutch college of general practitioners (first revision). Huisarts Wet. 2003;46:614-623.

8. Fernandez A, Haro JM, Martinez-Alonso M, et al. Treatment adequacy for anxiety and depressive disorders in six European countries. Br J Psychiatry. 2007;190:172-173.

9. Stein MB, Sherbourne CD, Craske MG, et al. Quality of care for primary care patients with anxiety disorders. Am J Psychiatry. 2004;161:2230- 2237.

10. Wang PS, Berglund P, Kessler RC. Recent care of common mental disorders in the United States: prevalence and conformance with evidence- based recommendations. J Gen Intern Med. 2000;15:284 292.

11. Wang PS, Lane M, Olfson M, et al. Twelve-month use of mental health services in the United States: results from the National Comorbidity Survey Replication. Arch Gen Psychiatry. 2005;62:629-640.

12. Wang PS, Aguilar-Gaxiola S, Alonso J, et al. Use of mental health services for anxiety, mood, and substance disorders in 17 countries in the WHO world mental health surveys. Lancet. 2007;370:841- 850.

13. Young AS, Klap R, Sherbourne CD, et al. The quality of care for depressive and anxiety disorders in the United States. Arch Gen Psychiatry. 2001;58:55- 61.

14. Smolders M, Laurant M, Verhaak $P$, et al. Adherence to evidence-based guidelines for depression and anxiety disorders is associated with recording of the diagnosis. Gen Hosp Psychiatry. 2009;31:460-469.

15. Andrews G, Issakidis C, Sanderson K, et al. Utilising survey data to inform public policy: comparison of the cost-effectiveness of treatment of ten mental disorders. Br J Psychiatry. 2004;184:526 -533.

16. Cabana MD, Rand CS, Powe NR, et al. Why don't physicians followclinical practice guidelines? A framework for improvement. JAMA 1999;282:1458-1465.

17. Osborn EH, Bird JA, McPhee SJ, et al. Cancer screening by primary care physicians. Can we explain the differences? J Fam Pract. 1991;32:465- 471.

18. Larme AC, Pugh JA. Attitudes of primary care providers toward diabetes: barriers to guideline implementation. Diabetes Care. 1998;21:1391- 1396.

19. Ward MM, Vaughn TE, Uden-Holman T, et al. Physician knowledge, attitudes and practices regarding a widely implemented guideline. J Eval Clin Pract. 2002;8:155-162.

20. Solomon DH, Brookhart MA, Gandhi TK, et al. Adherence with osteoporosis practice guidelines: a multilevel analysis of patient, physician, and practice setting characteristics. Am J Med. 2004;117:919 924.

21. Brownson RC, Davis JR, Simms SG, et al. Cancer control knowledge and priorities among primary care physicians. J Cancer Educ. 1993;8: 35-41.

22. Triezenberg DJ, Smith MA, Holmes TM. Cancer screening and detection in family practice: a MIRNET study. J Fam Pract. 1995;40:27-33.

23. Hargraves JL, Palmer RH, Orav EJ, et al. Practice characteristics and performance of primary care practitioners. Med Care. 1996;34(suppl 9): SS67-SS76.

24. Penninx BW, Beekman AT, Smit JH, et al. The Netherlands Study of Depression and Anxiety (NESDA): rationale, objectives and methods.

Int J Methods Psychiatr Res. 2008;17:121-140.

25. World Health Organization. Composite International Diagnostic Interview, Core Version 2.1: Interviewer's Manual. Sydney, Australia: World Health Organization; 1997.

26. Grol R, Thomas S, Roberts R. Development and implementation of guidelines for family practice: lessons from The Netherlands. J Fam Pract. 1995;40:435- 439.

27. Lamberts H, Wood M. International Classification of Primary Care (ICPC). Oxford, United Kingdom: Oxford University Press; 1990.

28. Meadows G, Harvey C, Fossey E, et al. Assessing perceived need for mental health care in a community survey: development of the Perceived Need for Care Questionnaire (PNCQ). Soc Psychiatry Psychiatr Epidemiol. 2000;35:427- 435. 
Smolders, M., Laurant, M., Verhaak, P., Prins, M., Marwijk, H. van, Penninx, B., Wensing, M., Grol, R. Which7 physician and practice characteristics are associated with adherence to evidence-based guidelines for 1 ( depressive and anxiety disorders? Medical Care: 2010, 48(3), 240-248

29. van den Hombergh $P$, Grol R, van den Hoogrn $\mathrm{HJ}$, et al. Assessment of management in general practice: validation of a practice visit method. Br J Gen Pract. 1998;48:1743-1750.

30. Botega N, Blizard R, Wilkinson GM. General practitioners and depression — first use of the depression attitude questionnaire. Int J Methods Psychiatr Res. 1992;4:169 -180.

31. McCall L, Clarke DM, Rowley G. A questionnaire to measure general practitioners' attitudes to their role in the management of patients with depression and anxiety. Aust Fam Physician. 2002;31:299 -303.

32. Haddad M, Walters $P$, Tylee A. District nursing staff and depression: a psychometric evaluation of depression attitude questionnaire findings. Int J Nurs Stud. 2007;44:447-456.

33. Peters MA, Harmsen M, Laurant MG, et al. Room for Improvement? Barriers and Opportunities for Improving Patient Care. Nijmegen, The Netherlands: Centre for Quality of Care Research; 2003.

34. Schaufeli WB, van Dierendonk D. UBOS (Utrechtse Burnout Schaal) Handleiding. Lisse, The Netherlands: Swets and Zeitlinger; 2000.

35. Rush AJ, Gullion CM, Basco MR, et al. The Inventory of Depressive Symptomatology (IDS): psychometric properties. Psychol Med. 1996; 26:477- 486.

36. Beck AT, Epstein N, Brown G, et al. An inventory for measuring clinical anxiety: psychometric properties. J Consult Clin Psychol. 1988;56:893- 897.

37. Snijders TA, Bosker RJ. Multilevel Analysis: An Introduction to Basic and Advanced Multilevel Modeling. London, United Kingdom: Sage; 1999.

38. Mosca L, Linfante AH, Benjamin EJ, et al. National study of physician awareness and adherence to cardiovascular disease prevention guidelines. Circulation. 2005;111:499 -510.

39. Gilbody S, Whitty P, Grimshaw J, et al. Educational and organizational interventions to improve the management of depression in primary care: a systematic review. JAMA. 2003;289:3145-3151.

40. Gilbody S, Bower P, Fletcher J, et al. Collaborative care for depression: a cumulative meta-analysis and review of longer-term outcomes. Arch Intern Med. 2006;166:2314 -2321.

41. Heideman J, van Rijswijk E, van Lin N, et al. Interventions to improve management of anxiety disorders in general practice: a systematic review. Br J Gen Pract. 2005;55:867- 874.

42. Smolders $M$, Laurant $M$, Roberge $P$, et al. Knowledge transfer and improvement of primary and ambulatory care for patients with anxiety. Can J Psychiatry. 2008;53:277-293.

43. Hearld D, Alexander J, Fraser I, et al. How do hospital organizational structure and processes affect quality of care? A critical review of research methods. Med Care Res Rev. 2008;65:259 -299.

44. Grol R, Grimshaw J. From best evidence to best practice: effective implementation of change in patients' care. Lancet. 2003;362:1225- 1230.

45. Grol R, Wensing M, Eccles M. Improving Patient Care. The Implementation of Change in Clinical Practice. Oxford, United Kingdom: Elsevier; 2005.

46. Harkness EF, Bower PJ. On-site mental health workers delivering psychological therapy and psychosocial interventions to patients in primary care: effects on the professional practice of primary care providers. Cochrane Database Syst Rev. 2008:CD000532.

47. Sol BG, van der Bijl J, Banga J, et al. Vascular risk management through nurse-led self-management programs. J Vasc Nurs. 2005;23:20 -24.

48. Wagner EH, Grothaus LC, Sandhu N, et al. Chronic care clinics for diabetes in primary care. Diabetes Care. 2001;24:695-700.

49. Laurant M, Reeves D, Hermens R, et al. Substitution of doctors by nurses in primary care. Cochrane Database Syst Rev. 2004:CD001271.

50. Laurant M, Harmsen M, Wollersheim H, et al. The impact of nonphysician clinicians: do they improve the quality and cost-effectiveness of healthcare services? Med Care Res Rev. 2009;66:36S- 89S.

51. Terluin B, van Marwijk H, Ader H, et al. The Four-Dimensional Symptom Questionnaire (4DSQ): a validation study of a multidimensional self-report questionnaire to assess distress, depression, anxiety and somatization. BMC Psychiatry. 2006;6:34.

52. Nutting PA, Rost K, Smith J, et al. Competing demands from physical problems: effect on initiating and completing depression care over 6 months. Arch Fam Med. 2000;9:1059-1064. 
Smolders, M., Laurant, M., Verhaak, P., Prins, M., Marwijk, H. van, Penninx, B., Wensing, M., Grol, R. Which physician and practice characteristics are associated with adherence to evidence-based guidelines for depressive and anxiety disorders? Medical Care: 2010, 48(3), 240-248

\section{FIGURE AND TABLES}

Figure 1. Recruitment flow of Netherlands Study of Depression and Anxiety respondents in the primary care setting. *Current _ presence during last 6 months; noncurrent _ presence before last 6 months, subthreshold symptoms defined as screen-positives or having a minor depression according to the composite interview diagnostic instrument interview.

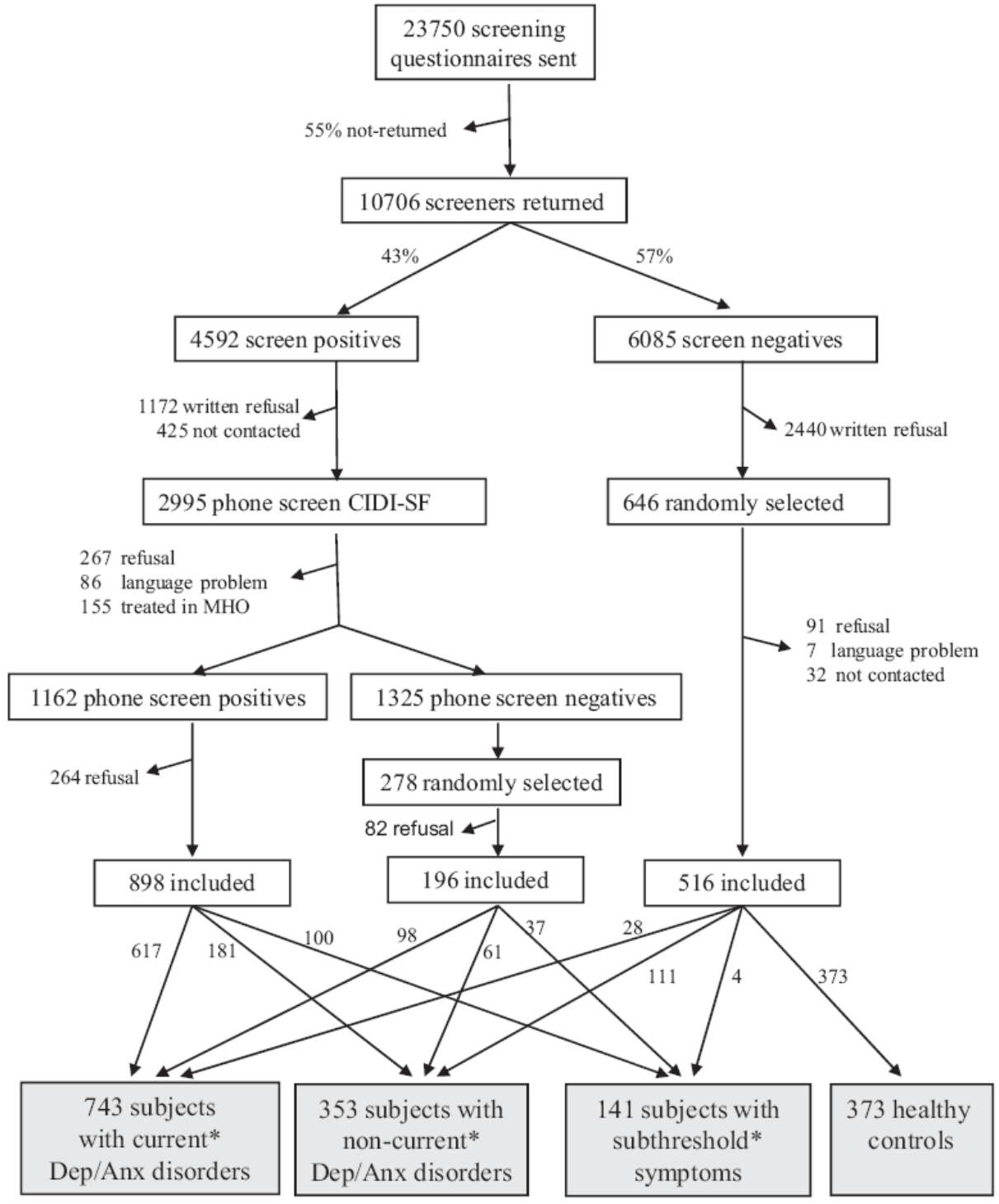

Recruitment flow of NESDA respondents in the primary care setting

* Current $=$ presence during last 6 months, non-current = presence before last 6 months, subthreshold symptoms defined as screenpositives or having a minor depression according to the CIDI interview 
Smolders, M., Laurant, M., Verhaak, P., Prins, M., Marwijk, H. van, Penninx, B., Wensing, M., Grol, R. Which physician and practice characteristics are associated with adherence to evidence-based guidelines for depressive and anxiety disorders? Medical Care: 2010, 48(3), 240-248

\begin{tabular}{ll}
\hline TABLE 1. Characteristics of the Patients $(\mathrm{n}=665)$ \\
\hline Age & \\
$\quad$ Mean (SD yr) & $44.9(12.0)$ \\
Gender & $201(30.2 \%)$ \\
$\quad$ Male & $464(69.8 \%)$ \\
Female & \\
CIDI diagnosis & $140(21.1 \%)$ \\
$\quad$ Current depressive disorder & $270(40.6 \%)$ \\
Current anxiety disorder & $255(38.3 \%)$ \\
Comorbid current depressive and anxiety disorder & $267(40.2 \%)$ \\
Overall guideline adherence & $107(16.1 \%)$ \\
Appropriate nonpharmacological treatment & $126(18.9 \%)$ \\
Appropriate pharmacological treatment & $156(23.5 \%)$ \\
Referral to specialized mental health care &
\end{tabular}

TABLE 2. Descriptive Characteristics of Practices $(n=21)$

\begin{tabular}{lc}
\hline & $\begin{array}{c}\text { Mean (SD) or } \\
\text { Number (\%) }\end{array}$ \\
\hline Practice type (\%) & \\
$\quad$ Solo (1 GP) & $3(14.3 \%)$ \\
$\quad$ Group ( $\geq 2$ GPs) & $12(57.1 \%)$ \\
$\quad$ Health centre (employing both GPs and other health & $6(28.6 \%)$ \\
$\quad$ care professionals) & \\
No. GPs in the practice, mean no. GPs (SD) & $3.4(2.0)$ \\
Health care professionals working in the practice (\%) & \\
$\quad$ Practice assistant & $21(100.0 \%)$ \\
$\quad$ Practice nurse & $16(76.2 \%)$ \\
$\quad$ Nurse practitioner & $1(4.8 \%)$ \\
$\quad$ Mental health nurse & $12(57.1 \%)$ \\
$\quad$ Social worker & $5(23.8 \%)$ \\
$\quad$ Psychologist & $6(28.6 \%)$ \\
No. patients cared for per GP (SD) & $1646(559.4)$ \\
Size of practice population, mean no. patients (SD) & $5013(3071.2)$ \\
\hline
\end{tabular}


Smolders, M., Laurant, M., Verhaak, P., Prins, M., Marwijk, H. van, Penninx, B., Wensing, M., Grol, R. Which physician and practice characteristics are associated with adherence to evidence-based guidelines for depressive and anxiety disorders? Medical Care: 2010, 48(3), 240-248

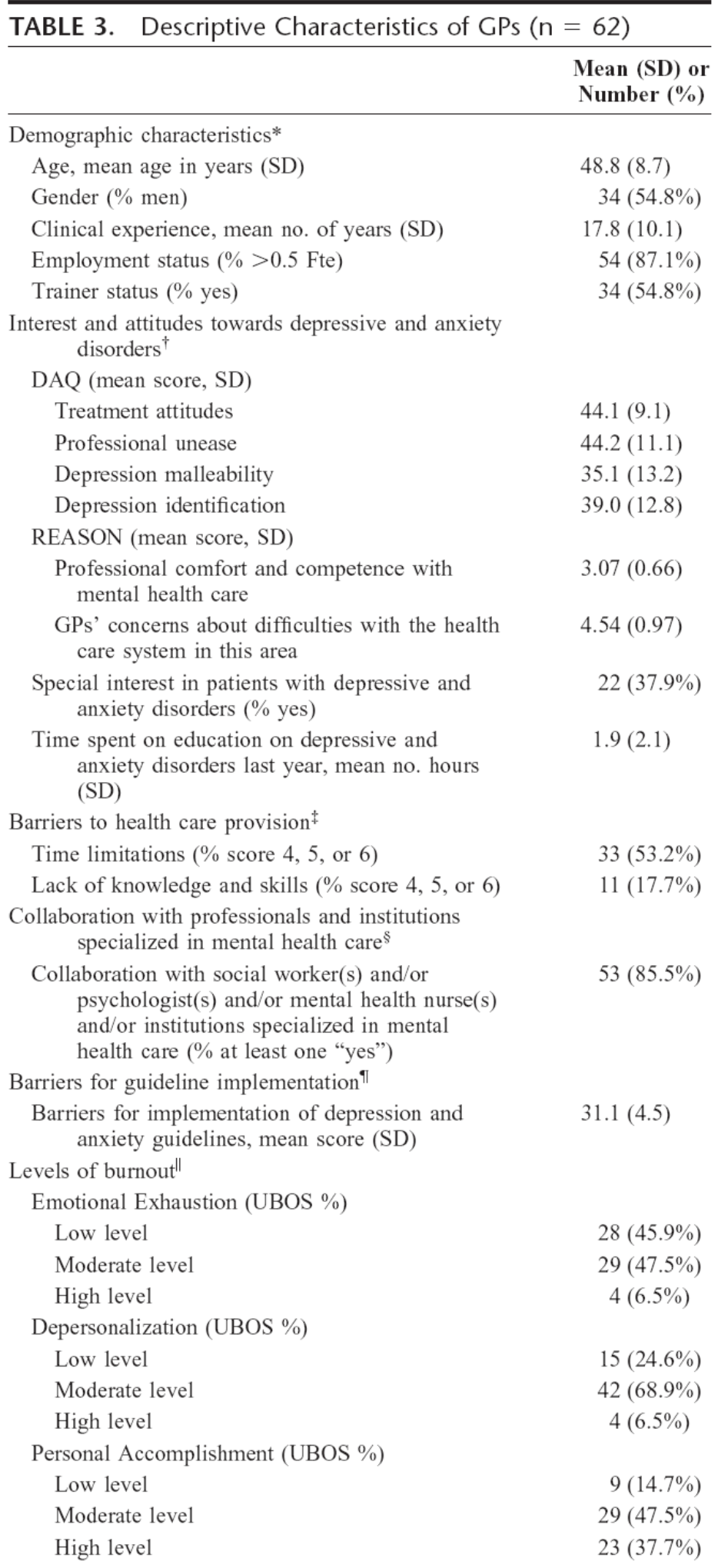

*Various questions.

${ }^{\dagger}$ Depression Attitude Questionnaire (visual analogue scale varying from strongly disagree $[0 \mathrm{~mm}]$ to strongly agree $[100 \mathrm{~mm}])+$ REASON (7-point Likert scale [1: very strongly agree-7: very strongly disagree]) +2 additional items.

Two-item questionnaire (6-point Likert scale [1: to no extent-6: to a very great extent]).

${ }^{\S}$ Four-item questionnaire (dichotomous items)

"Thirteen-item questionnaire (5-point Likert scale [1: fully disagree-5: fully agree]).

"Utrecht Burn-Out Scale for the contactual professions (7-point Likert scale [0: never-6: always]). 
Smolders, M., Laurant, M., Verhaak, P., Prins, M., Marwijk, H. van, Penninx, B., Wensing, M., Grol, R. Which physician and practice characteristics are associated with adherence to evidence-based guidelines for depressive and anxiety disorders? Medical Care: 2010, 48(3), 240-248

TABLE 4. Univariate and Multivariate Associations Between Practice and Professional Characteristics* on the One Hand, and Overall Guideline Adherence and Various Treatment Options on the Other

\begin{tabular}{|c|c|c|c|c|}
\hline & \multirow{2}{*}{$\begin{array}{c}\text { Univariate } \\
\text { Associations } \\
P\end{array}$} & \multirow{2}{*}{$\begin{array}{c}\text { Multivariate } \\
\text { Associations } \\
P\end{array}$} & \multicolumn{2}{|c|}{$\begin{array}{c}\text { Multivariate } \\
\text { Associations } \\
\text { (Final Model) }\end{array}$} \\
\hline & & & OR $(95 \% \mathrm{CI})$ & $P$ \\
\hline \multicolumn{5}{|l|}{ Outcome: overall guideline adherence } \\
\hline GPs' confidence in depression identification & 0.07 & 0.05 & $0.99(0.99-0.99)$ & 0.02 \\
\hline $\begin{array}{l}\text { Collaboration with professionals and institutions } \\
\text { specialized in }\end{array}$ & 0.02 & 0.06 & NA & NA \\
\hline \multicolumn{5}{|l|}{ Mental health care } \\
\hline Age & 0.10 & 0.11 & NA & NA \\
\hline Barriers for guideline implementation & 0.05 & 0.10 & NA & NA \\
\hline \multicolumn{5}{|l|}{ Outcome: appropriate nonpharmacological treatment } \\
\hline GPs' confidence in depression identification & 0.06 & 0.16 & NA & NA \\
\hline Personal accomplishment & 0.02 & 0.07 & NA & NA \\
\hline $\begin{array}{l}\text { GPs' concerns about difficulties with the health care } \\
\text { system }\end{array}$ & 0.10 & 0.14 & NA & NA \\
\hline Time limitations & 0.01 & $<0.01$ & $1.31(1.06-1.62)$ & 0.01 \\
\hline \multicolumn{5}{|l|}{ Outcome: appropriate pharmacological treatment } \\
\hline Size of practice population & 0.09 & 0.08 & NA & NA \\
\hline Employment status & 0.08 & 0.14 & NA & NA \\
\hline \multicolumn{5}{|l|}{ Outcome: referral to specialized mental health care } \\
\hline Age & 0.07 & 0.40 & NA & NA \\
\hline Barriers for guideline implementation & 0.05 & 0.01 & $0.92(0.87-0.98)$ & $<0.01$ \\
\hline GPs' confidence in depression identification & $<0.01$ & 0.02 & $0.99(0.98-0.99)$ & $<0.01$ \\
\hline $\begin{array}{l}\text { Special interest in patients with depressive and } \\
\text { anxiety disorders }\end{array}$ & 0.05 & 0.15 & NA & NA \\
\hline Personal accomplishment & 0.07 & 0.59 & NA & NA \\
\hline
\end{tabular}

